\title{
The safety of anaesthetists and intensivists during the first COVID-19 surge supports extension of use of airborne protection PPE to ward staff
}

\author{
Authors: Tim M Cook, ${ }^{A}$ Kariem El-Boghdadly, ${ }^{A, B}$ Jules Brown ${ }^{C}$ and Anthony E Pickering ${ }^{D}$
}

\begin{abstract}
A key controversy in the COVID-19 pandemic has been over staff safety in health and social care settings. Anaesthetists and intensivists were anticipated to be at the highest risk of work-related infection due to involvement in airway management and management of critical illness and therefore wear the highest levels of personal protective equipment (PPE) in the hospital. However, the data clearly show that those working in anaesthesia and critical care settings are at lower risk of infection, harm and death from COVID-19 than colleagues working on the wards. The observed safety of anaesthetists and intensivists and increased risk to those in other patient-facing roles has implications for transmissionbased infection control precautions. The precautionary principle supports extending training in and use of airborne precaution PPE to all staff working in patient-facing roles who have close contact with coughing patients. This will both reduce their risk of contracting COVID-19, maintain services and reduce nosocomial transmission to vulnerable patients. The emergence of a new variant of the SARS-CoV-2 virus with significantly higher transmissibility creates urgency to addressing this matter.
\end{abstract}

KEYWORDS: COVID-19, safety, personal protective equipment

DOI: $10.7861 /$ clinmed.2020-0983

\section{Introduction}

The phrase 'coughs and sneezes spread diseases' originates from an American public health campaign during the 1918 influenza pandemic. It remains just as true and relevant in the current COVID-19 pandemic and encapsulates why personal protective equipment (PPE) is such a key issue.

Authors: ${ }^{A}$ consultant in anaesthesia and intensive care medicine, Royal United Hospitals NHS Foundation Trust; ${ }^{\mathrm{B}}$ consultant in Anaesthesia, Guy's and St Thomas' NHS Foundation Trust; 'Consultant in anaesthesia and intensive care medicine, North Bristol NHS Trust; Dprofessor of neuroscience and anaesthesia, School of Physiology, Pharmacology and Neuroscience, University of Bristol
Here, we review the current evidence on COVID-19-related mortality in different healthcare professional groups and our understanding of how COVID-19 is transmitted and ask whether the current guidelines on use of PPE should change. The emergence of a new variant of the SARS-CoV-2 virus with significantly higher transmissibility creates urgency to addressing this matter.

Principles behind the use of personal protective equipment for COVID-19

A wealth of data show that coughing and sneezing (and loud talking and singing) create a directional plume of potentially infectious airborne particles.' False dichotomies have arisen over the designation of these particles into droplets ( $>10 \mu \mathrm{m}$ diameter) and aerosols $(<10 \mu \mathrm{m})$ and over which is the principal source of SARS-CoV-2 transmission. Importantly, smaller particles may remain suspended in the air for prolonged periods and circumvent a loose-fitting mask or visor.

PPE is designed to protect the wearer against infection and what PPE should be worn by healthcare workers is intellectually uncomplicated: simply match the type PPE to the mode of viral transmission. ${ }^{2}$ So where contact or droplet or aerosol are thought to be the main mode of transmission the PPE worn should be 'contact precaution,' 'droplet precaution' or 'airborne precaution', respectively. The stance adopted by the UK public health agencies is that when certain medical procedures, designated 'aerosol generating procedures' (AGPs) are performed, airborne precaution PPE should be worn which, in addition to gloves and eye protection, includes a water-repellent gown that covers the arms and a filtering face piece/respirator - FFP3 or N95. At other times, droplet precaution PPE, which comprises a simple apron and a fluid resistant surgical mask, is deemed sufficient.? Importantly, a surgical face mask may provide a degree of protection against inhalation of particulate matter, but that is not its main purpose - which is to protect those around the wearer. ${ }^{1,2}$ In contrast, a correctly fitted valveless filtering face piece protects protect those around the wearer and also the wearer, filtering out $>99 \%$ of particulate matter during inhalation. ${ }^{3}$

In the UK, the designated list of AGPs includes almost all airway management procedures undertaken by anaesthetists and intensivists who are therefore judged to be at high risk of infection and are also more likely to wear airborne precaution PPE. However, the available evidence contradicts this stance. 


\section{Data on healthcare worker risk}

UK patient-facing healthcare workers are at increased risk of SARSCoV-2 infection and of death from COVID-19. Data consistently show increased rates of healthcare worker infection compared to their community, with rates ranging from two-fold to four-fold higher. ${ }^{4-10}$ Increased hospital prevalence is often caused by wardlevel outbreaks, ${ }^{711}$ can lead to nosocomial infection and has on occasion led to closure of whole hospitals. Counterintuitively, there is increasing evidence that compared to other hospital workers, those working in anaesthesia and critical care are at reduced risk of SARS-CoV-2 infection or death from COVID-19. In the SAFER study, intensivists had the lowest rates of all specialties both for SARS-CoV-2 infection and seroconversion. ${ }^{7}$ Those working in anaesthesia and critical care have similarly been found to be at low risk of infection in studies from Oxford, ${ }^{7}$ Leicester, ${ }^{8}$ Birmingham ${ }^{10}$ and Scotland. ${ }^{12}$ The groups of individuals who appear to be most affected are those exposed to infected patients on the wards: that is nurses (especially those working in respiratory care), junior doctors, housekeeping staff, and acute and respiratory physicians. ${ }^{6-8,10,12}$

A national study of $>150,000$ healthcare workers throughout Scotland reported a three-fold increase in hospital admissions with COVID-19 among patient-facing healthcare workers compared to healthcare workers in non-patient-facing roles and the general population. The extent of the increased risk had increased during the pandemic. ${ }^{10}$ Those working in critical care were half as likely to be admitted as those working in 'front door' roles and were less likely to be admitted than household contacts of frontline healthcare workers.

In May, the deaths of 31 UK doctors from COVID-19 were reported: ${ }^{13}$ although anaesthetists and intensivists account for more than one in nine of all doctors, none from these specialties were among these fatalities. Those who work closely with anaesthetists and intensivists, and who also wear airborne PPE, are also protected: of 166 UK healthcare worker deaths only one involved a healthcare worker who worked alongside anaesthetists or intensivists. ${ }^{13}$ The international literature is in line with this: while deaths from COVID-19 among anaesthetists and intensivists are not unknown, they are fewer than expected, with more deaths among general practitioners and emergency and respiratory physicians. ${ }^{14}$ In November 2020, the first death of a UK anaesthetist from COVID-19 was announced. Despite this sad news, a numerical analysis confirms anaesthetists and intensivists are underrepresented compared to other specialties in terms of risk of death from COVID-19.15

\section{Explaining differences in healthcare worker risk}

How then do we account for contradictory observation that those expected to be at highest risk appear to be the safest? There are several explanations. Perhaps the most obvious is that those undertaking AGPs use higher performing PPE. Anaesthetists and intensivists also generally work in well-ventilated locations. Anaesthetists and intensivists are generally expert at infection control precautions and use of PPE in order to maintain surgical sterility in operating theatres or to reduce cross-infection in intensive care, so it is not a major change of behaviour from protecting the patient to protecting themselves.

Recent data have raised questions as to whether procedures currently classified as AGPs actually generate aerosols, including tracheal intubation and extubation ${ }^{16}$, non-invasive ventilation and high-flow nasal oxygen. ${ }^{17}$ Importantly these studies do confirm significant aerosol generation during coughing, even when this is done by a healthy volunteer. ${ }^{16,17}$ In contrast to other coronaviruses causing severe acute respiratory distress, it is notable that in patients with COVID-19 viral secretion and infectious risk usually peaks in the 2 days before symptoms and the 5 days afterwards, with infectivity most commonly dissipated by day $9-10$ of symptoms. ${ }^{18}$ Of note, critical illness from COVID-19 usually presents 10-12 days after symptom onset and it is plausible that infectious risk may be low at this time. ${ }^{18}$ Finally, coughing and sneezing events (aerosol generating events) on wards occur much more commonly than do AGPs during interventional airway management, so the duration of aerosol exposure may be lower in theatres and in critical care than on the wards.

The explanation for why anaesthetists, intensivists and those they work with are at lower risk of SARS-CoV-2 transmission than those in patient-facing roles on the wards is uncertain, but the finding is consistent and from multiple sources. In keeping with the idea that use of high quality of PPE is a critical factor is the finding by Nguyen and colleagues that healthcare worker infection was strongly associated with caring for COVID-19 infected patients and with reuse (without sterilisation) or lack of access to PPE, such that the combination of the two led to a more than five-fold increase in nosocomial infection rates. ${ }^{9}$

What then should we learn from the observations in the first wave of the pandemic and should we alter our behaviour now? Given the current uncertainty, we believe that those working in operating theatres and critical care should continue doing broadly what they are already doing. However, without changes to practice we can anticipate that in future surges, and particularly as hospitals strive to maintain both COVID-19 and non-COVID-19 work, COVID-19 outbreaks in hospitals will continue and infected staff will, often asymptomatic and unknowingly, pass it on to other staff and to patients..$^{11,19}$ Such transmission has important implications for patients: both surgical and medical patients who acquire COVID-19 while in hospital have a mortality rate of more than $20 \% .{ }^{20}$ Further, staff infection not only increases the risk of harm to these staff and other patients but it is disruptive to services and may lead to their interruption.

We should change policy to ensure that, during the pandemic, as a minimum all staff coming into close contact with coughing patients should be wearing airborne precaution PPE that includes a protective facemask/respirator, such as an FFP3 or N95 mask.

\section{Conclusion: ward staff need airborne protection PPE}

On the wards, patients will continue to cough and sneeze and undoubtedly expel droplets and aerosols that travel significant distances, patients' masks will slip, and patients are more likely to be in the phase of the illness when viral secretion is maximal. Hospital-based studies consistently show that 'frontline' and 'front door' staff, including acute care physicians and others managing these patients on open wards, are among the highest risk groups for transmission and harm from nosocomial COVID-19. Frontline healthcare workers are at significantly increased risk of harm, including death, from COVID-19 compared to the general public and this disproportionately affects those working in close patient contact. ${ }^{21}$ Employers have a legal responsibility to protect staff from harm and a precautionary solution, based on the above knowledge, would be to dramatically increase the use of airborne 
precaution PPE and training in its correct use. Most precautionary would be to apply this to all clinical and non-clinical staff coming into close contact (eg 2 metres) with patients who have or are at risk of SARS-CoV-2 infection, and this would be in line with guidance from the Centers for Disease Control and Prevention in the USA. ${ }^{22}$ A pragmatic and graded solution would be to apply it to all such patients who are coughing or sneezing and therefore known to be expelling aerosols. This will undoubtedly be a logistical challenge, but it is the right action to preserve hospital safety and efficacy. In recent months, the rate of nosocomial infections of staff and patients has risen, impacting on both staff safety and service delivery. In December 2020, the emergence of a SARS-CoV-2 variant, with significantly increased transmissibility, ${ }^{23}$ has increased transmission in the south east of the UK and is now the dominant strain in most parts of the country. This new variant both highlights the importance of this matter and creates urgency in addressing this risk.

\section{References}

1 Thomas JP, Srinivasan A, Wickramarachchi CS et al. Evaluating the national PPE guidance for NHS healthcare workers during the COVID-19 pandemic. Clin Med 2020;20:242-47.

2 Cook TM. Personal protective equipment during the coronavirus disease (COVID) 2019 pandemic - a narrative review. Anaesthesia 2020;75:920-7.

3 Ueki H, Furusawa Y, Iwatsuki $\mathrm{K}$ et al. Effectiveness of face masks in preventing airborne transmission of SARS-CoV-2. mSphere 2020:5:e00637-20.

4 Pouwels KB, House T, Robotham JV et al. Community prevalence of SARS-CoV-2 in England: Results from the ONS Coronavirus Infection Survey Pilot. www.medrxiv.org/content/10.1101/2020.07. 06.20147348v1 [Accessed 16 October 2020].

5 Ward H Cooke G, Atchison C et al. Declining prevalence of antibody positivity to SARS-CoV-2: a community study of 365,000 adults. www.medrxiv.org/content/10.1101/2020.10.26.20219725v1 [Accessed 16 October 2020].

6 Houlihan CF, Vora N, Byrne T et al. Pandemic peak SARS-CoV-2 infection and seroconversion rates in London frontline health-care workers. Lancet 2020;396:e6-e7.

7 Eyre DW, Lumley SF, O'Donnell D et al. Differential occupational risks to healthcare workers from SARS-CoV-2 observed during a prospective observational study. Elife 2020;9:e60675.

8 Martin CA, Patel P, Goss C et al. Demographic and occupational determinants of anti-SARS-CoV-2 IgG seropositivity in hospital staff. J Public Health 2020, in press (doi: 10.1093/pubmed/ fdaa199).

9 Nguyen LH, Drew DA, Graham MS et al. Risk of COVID-19 among front-line health-care workers and the general community: a prospective cohort study. Lancet Public Health 2020;5:e475-83.
10 Shah ASV, Wood R, Gribben C et al. Risk of hospital admission with coronavirus disease 2019 in healthcare workers and their households: nationwide linkage cohort study. BMJ 2020;371:m3582.

11 Rivett R, Sridhar S, Sparkes D et al. Screening of healthcare workers for SARS-CoV-2 highlights the role of asymptomatic carriage in COVID-19 transmission. elife 2020;9:e58728.

12 Shields A, Faustini SE, Perez-Toledo M et al. SARS-CoV-2 seroprevalence and asymptomatic viral carriage in healthcare workers: a cross-sectional study. Thorax 2020;75:1089-94.

13 Kearney L, Lennane S, Woodman E et al. At least 23 nationalities among NHS staff killed by covid. Health Service J 19 May 2020. www.hsj.co.uk/workforce/at-least-23-nationalities-among-nhs-staffkilled-by-covid/7027666.article [Accessed 16 October 2020].

14 Kursomovic E, Lennane S, Cook TM. COVID-19 associated mortality in health care workers: a call for UK-wide reporting registry. Anaesthesia 2020:75;989-92.

15 Cook TM, Lennane S. Occupational COVID-19 risk for anaesthesia and intensive care staff - low-risk specialties in a high-risk setting. Anaesthesia 2020, in press (doi: 10.1111/anae.15358).

16 Brown J, Gregson FKA, Shrimpton A et al. Comparative risk evaluation of aerosol generation by tracheal intubation and extubation. Anaesthesia 2020, in press (doi: 10.1111/anae.15292).

17 Gaeckle NT, Lee J, Park $Y$ et al. Aerosol generation from the respiratory tract with various modes of oxygen delivery. Am J Resp Crit Care Med 2020:202:1115-24.

18 Cevik M, Tate M, Lloyd O et al. SARS-CoV-2, SARS-CoV, and MERS-CoV viral load dynamics, duration of viral shedding, and infectiousness: a systematic review and meta-analysis. Lancet Microbe 2020, in press (doi: 10.1016/S2666-5247(20)30172-5).

19 Carter B, Collins JT, Barlow-Pay F et al. Nosocomial COVID-19 infection: examining the risk of mortality. The COPE-Nosocomial Study (COVID in Older PEople). J Hosp Infect 2020;106:376-84.

20 COVIDSurg Collaborative. Mortality and pulmonary complications in patients undergoing surgery with perioperative SARS-CoV-2 infection: an international cohort study. Lancet 2020;396:27-38.

21 Cook TM, Lennane S. Nurses, ambulance crews and support workers should get the Covid vaccine first. Health Service J, 31 December 2020. www.hsj.co.uk/coronavirus/nurses-ambulance-crews-andsupport-workers-should-get-the-covid-vaccine-first/7029239.article.

22 Centers for Disease Prevention and Control. Interim infection prevention and control recommendations for healthcare personnel during the coronavirus disease 2019 (COVID-19) pandemic. CDC, 2020. www.cdc.gov/coronavirus/2019-ncov/hcp/infection-controlrecommendations.html [Accessed 17 October 2020].

23 Wise J. Covid-19: New coronavirus variant is identified in UK. BMJ 2020;371:m4857371.

Address for correspondence: Prof Tim Cook, Royal United Hospitals, Combe Park, Bath, BA1 3NG, UK. Email: timcook007@gmail.com 